Kardiologe $2022 \cdot 16: 242-249$

https://doi.org/10.1007/s12181-022-00536-6

Angenommen: 24. Januar 2022

Online publiziert: 17. Februar 2022

๑) Der/die Autor(en) 2022

\section{Kontrastmittelinduzierte Nierenfunktionsstörungen gemessen am Serumcystatin C}

\author{
Ein Vergleich zwischen euvolämen und hypovolämen \\ Patienten nach Koronarangiographie
}

Farshad Moussazadeh Vahed · Hans-Joachim Trappe

Medizinische Klinik II (Schwerpunkte Kardiologie und Angiologie), Marien Hospital Herne

Universitätsklinik, Ruhr-Universität Bochum, Herne, Deutschland

\title{
Zusammenfassung
}

Zusammenfassung: Die Bedeutung der kontrastmittelinduzierten Nierenfunktionsstörung (CIN) ist unklar. Diese prospektive Studie untersucht den Einfluss der Hypovolämie auf die Cystatin-C-Werte nach Kontrastmittelexposition im Rahmen einer Herzkatheteruntersuchung.

Patienten und Methoden: Insgesamt wurden 103 Patienten, davon 49 euvolämische und 54 hypovolämische $(68,72 \pm 12,36$ Jahren, 63,10\% männlich) eingeschlossen. Der Volumenstatus wurde anhand ultraschallgestützter Bestimmung des Collapsibility Index der V. cava inferior bestimmt. Vor, am ersten und zweiten Tag nach der HKU wurden Cystatin-C-Werte bestimmt. Ein Anstieg um 10\% der Baseline wurde als CINB deklariert.

Ergebnisse: Bei 8 (16,33\%) euvolämen und 21 (38,89\%) hypovolämen Patienten erkrankten an einer CINB $(p=0,015)$. Das relative Risiko (RR) für eine CINB bei hypovolämen Patienten betrug 2,38 (95\%-KI=1,163-4,877) $(p=0,015)$. Vorhofflimmern (VHF) war ebenfalls ein Risikofaktor für eine CINB: RR $=2,488$ (95\%$\mathrm{KI}=1,373-4,508)(p=0,003) ; \mathrm{OR}=6,606(95 \%-\mathrm{KI}=1,909-22,861)$. Patienten mit einem $\mathrm{BMI}>30 \mathrm{~kg} / \mathrm{m}^{2}$ hatten ein erhöhtes Risiko, an einer CINB zu erkranken: $\mathrm{OR}=4,326$ $(95 \%-\mathrm{KI}=1,281-14,602)(p=0,018), \mathrm{RR}=2,166(95 \%-\mathrm{KI}=1,163-4,036)(p=0,014)$. Patienten, die Schleifendiuretika einnahmen, ebenfalls ein erhöhtes Risiko für eine CINB: $\mathrm{RR}=1,976(95 \%-\mathrm{KI}=1,056-3,699)(p=0,033)$. Die chronische Herzinsuffizienz (CHF) war ein prädiktiver Faktor für die CINB: OR=13,27 (95\%-KI=1,453-121,199) $(p=0,018)$.

Schlussfolgerungen: Definiert man die akute Nierenfunktionsstörung anhand einer Serumcystatin-C-Erhöhung $>10 \%$, weisen hypovoläme Patienten ein erhöhtes Risiko für eine CINB nach Kontrastmittelexposition auf als euvoläme Patienten. Zudem sind VHF, die CHF und die Einnahme von Diuretika mit einem erhöhten Risiko für ein CINB assoziiert.

\section{Schlüsselwörter}

Cystatin C · Kontrastmittelnephropathie · Kontrastmittel · Volumenstatus · Herzkatheteruntersuchung

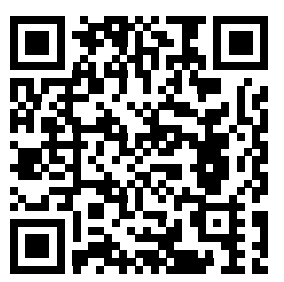

QR-Code scannen \& Beitrag online lesen
Es existiert eine Vielzahl an Studien zu der CIN, die zu widersprüchlichen Ergebnissen geführt haben. Es wird vermutet, dass Nierenschädigungen, die auf die Verabreichung von jodhaltigem Kontrastmittel (JKM) zurückzuführen sind, zu den häufigsten Nierenfunktionsstörungen gehören, die iatrogen verursacht werden [19].
Ergebnisse früherer Studien weisen darauf hin, dass die CIN mit einer erhöhten Morbidität und 1-Jahres-Mortalität einhergeht, den Krankenhausaufenthalt verlängert [25] und ein erhöhtes Risiko für ischämische Infarkte darstellt [4]. Bei nierengesunden Patienten ist die Inzidenz der CIN mit $<3 \%$ eher niedrig, kann jedoch 
Tab. 1 Ein- und Ausschlusskriterien

\section{Einschlusskriterien:}

Alter > 18 Jahre

Spontan atmende Patienten

HKU am Tag nach stationärer Krankenhausaufnahme normwertiges Krea im Serum vor HKU

(männliche Patienten $<1,1 \mathrm{mg} / \mathrm{dl}$, weibliche Patienten $<0,9 \mathrm{mg} / \mathrm{dl}$ )

\section{Ausschlusskriterien:}

Alter $<18$ Jahre

Erhöhtes Krea im Serum vor der HKU

(Männliche Patienten > 1,1 mg/dl, weibliche Patienten >0,9 mg/dl) hypervolämischer Hydratationszustand JKM-Exposition in den letzten 6 Monaten

Lungenödem

Unfähigkeit, die Atemmanöver durchzuführen

\begin{tabular}{|c|c|c|c|c|}
\hline Charakteristika & CINB (-) & CINB (+) & Summe & $p$-Wert \\
\hline Euvoläm, $n(\%)$ & $41(38,81)$ & $8(7,77)$ & $49(47,57)$ & - \\
\hline Hypovoläm, $n(\%)$ & $33(32,04)$ & $21(20,39)$ & $54(52,43)$ & 0,015 \\
\hline Mittleres Alter (Jahre) & $66,70 \pm 12,03$ & $68,72 \pm 12,36$ & - & 0,278 \\
\hline Männer, $n(\%)$ & $49(47,57)$ & $16(24,62)$ & $65(63,11)$ & n.s. \\
\hline Frauen, $n(\%)$ & $25(24,27)$ & $13(34,21)$ & $38(36,89)$ & 0,335 \\
\hline \multicolumn{5}{|l|}{ Komorbiditäten } \\
\hline aHtn & $75(83,33 \%)$ & $15(16,66 \%)$ & $90(90,90 \%)$ & n.s. \\
\hline $\mathrm{CHF}$ & $55(53,40 \%)$ & $28(27,18 \%)$ & $83(80,58 \%)$ & 0,011 \\
\hline KHK & $48(46,60 \%)$ & $15(14,56 \%)$ & $63(61,16 \%)$ & 0,475 \\
\hline VHF & $16(15,53 \%)$ & $15(14,56 \%)$ & $31(29,13 \%)$ & 0,004 \\
\hline DM Typ II & $14(13,59 \%)$ & $4(3,88 \%)$ & $18(17,47 \%)$ & 0,994 \\
\hline $\begin{array}{l}\text { Adipositas } \\
\left(\mathrm{BMI}>30 \mathrm{~kg} / \mathrm{m}^{2}\right)\end{array}$ & $17(16,50 \%)$ & $13(12,62 \%)$ & $30(29,13 \%)$ & 0,018 \\
\hline \multicolumn{5}{|l|}{ Medikamente } \\
\hline Schleifendiuretika & $26(25,24 \%)$ & $17(16,50 \%)$ & $43(41,74 \%)$ & 0,044 \\
\hline NSAR & $12(11,65 \%)$ & $4(3,88 \%)$ & $16(15,53 \%)$ & 1,000 \\
\hline Metformin & $8(7,77 \%)$ & $4(3,88 \%)$ & $12(11,65 \%)$ & 0,735 \\
\hline Statine & $47(45,63 \%)$ & $18(17,48 \%)$ & $65(63,11 \%)$ & 1,000 \\
\hline \multicolumn{5}{|l|}{ CIN Tag 1} \\
\hline $\mathrm{CIN}(+)$ & $2(1,94 \%)$ & $2(1,94 \%)$ & $4(3,88 \%)$ & - \\
\hline CIN (-) & $81(78,64 \%)$ & $18(17,47 \%)$ & $98(95,14 \%)$ & 0,168 \\
\hline \multicolumn{5}{|l|}{ CIN Tag 2} \\
\hline $\mathrm{CIN}(+)$ & $8(7,77 \%)$ & $4(3,88 \%)$ & $12(11,65 \%)$ & - \\
\hline CIN (-) & $86(83,49 \%)$ & $5(4,85 \%)$ & $91(88,35 \%)$ & 0,010 \\
\hline
\end{tabular}

abhängig von Risikofaktoren und Population auf bis zu $34 \%$ ansteigen [17, 21]. Dies lässt sich auch dadurch erklären, dass die Definition einer CIN nicht einheitlich geregelt ist.

Eine alternative Definition der CIN, ist durch den Anstieg des CysC-Wertes um $\geq 10 \%$ des Ausgangswertes im Zeitraum von $48 \mathrm{~h}$ nach Applikation von JKM gekennzeichnet [3] und wird im Folgenden als CINB bezeichnet. Wegen der stabilen Sekretionsrate von CysC, die im Gegensatz zu Krea unabhängig von der Nahrung oder dem Stoffwechsel der Musku-

\section{Material und Methoden}

\section{Patienten}

Bei dieser Studie handelt es sich um eine prospektiv monozentrische Beobachtungsstudie. Durchgeführt wurde die Studie zwischen dem 01.06.2017 und dem 30.11.2018. Insgesamt wurden 103 Patienten, bei denen am Tag nach der stationären Aufnahme eine elektive Herzkatheteruntersuchung (HKU) durchgeführt wurde, in diese Studie eingeschlossen. Einund Ausschlusskriterien sind in $\mathbf{0}$ Tab. 1 zusammengefasst.

\section{Methoden}

Sowohl vor als auch am ersten und zweiten Tag nach der HKU wurde bei allen Patienten Blut entnommen und die CysC ( $\mathrm{mg} / \mathrm{l})$ und Krea (mg/dl) -Werte im Serum bestimmt. Am Tag der HKU wurde im Herzkatheterlabor der Hydratationszustand aller Studienteilnehmer anhand des Diameters der V. cava inferior (DVCl) sonographisch mit dem Ultraschallgerät GE VIVID S6 (General Electric Healthcare Company, Chicago, IL, USA) ermittelt [1, 2, 23]. Nach Darstellung der V. cava inferior ( $\mathrm{VCl}$ ) in subxiphoidaler Sagittalebene in Rückenlage wurde der Patient gebeten, maximal ein- (max Insp) und maximal auszuatmen (max Exsp). Die Untersuchungen wurden von einem Facharzt für Innere Medizin durchgeführt, wobei die Messungen 3-mal wiederholt wurden. Ein anderer Facharzt (Innere Medizin und Kardiologie) kontrollierte und validierte die Befunde. Zwischen jeder Messung wurde eine Pause von $1 \mathrm{~min}$ eingelegt. Die Messergebnisse (in $\mathrm{cm}$ ) wurden gemittelt und anschließend in die Formel zur Ermittlung des Vena cava inferior Collapsibility Index (VCICl) eingefügt: $\mathrm{VICl}(\%)=((D V C I$ max Exsp-DVCImin Insp) / (DVCI max Ex$s p) \times 100 \%$ ). Bei einem $\mathrm{VCICl}$ von $20-50 \%$ wurde von einem euvolämen (ev) und bei einem $\mathrm{Cl}$ von $>50 \%$ von einem hypovolämen (hv) Hydratationszustand ausgegangen ([11, 18]; - Abb. 1 und 2). Da der Diameter der V. cava inferior zwar ein klinisch gebräuchlicher, aber doch fehlerbehafteter Parameter ist, sollte der Vena cava inferior Collapsibility Index ( $\mathrm{VCICl}$ ) besser verwendet werden. 


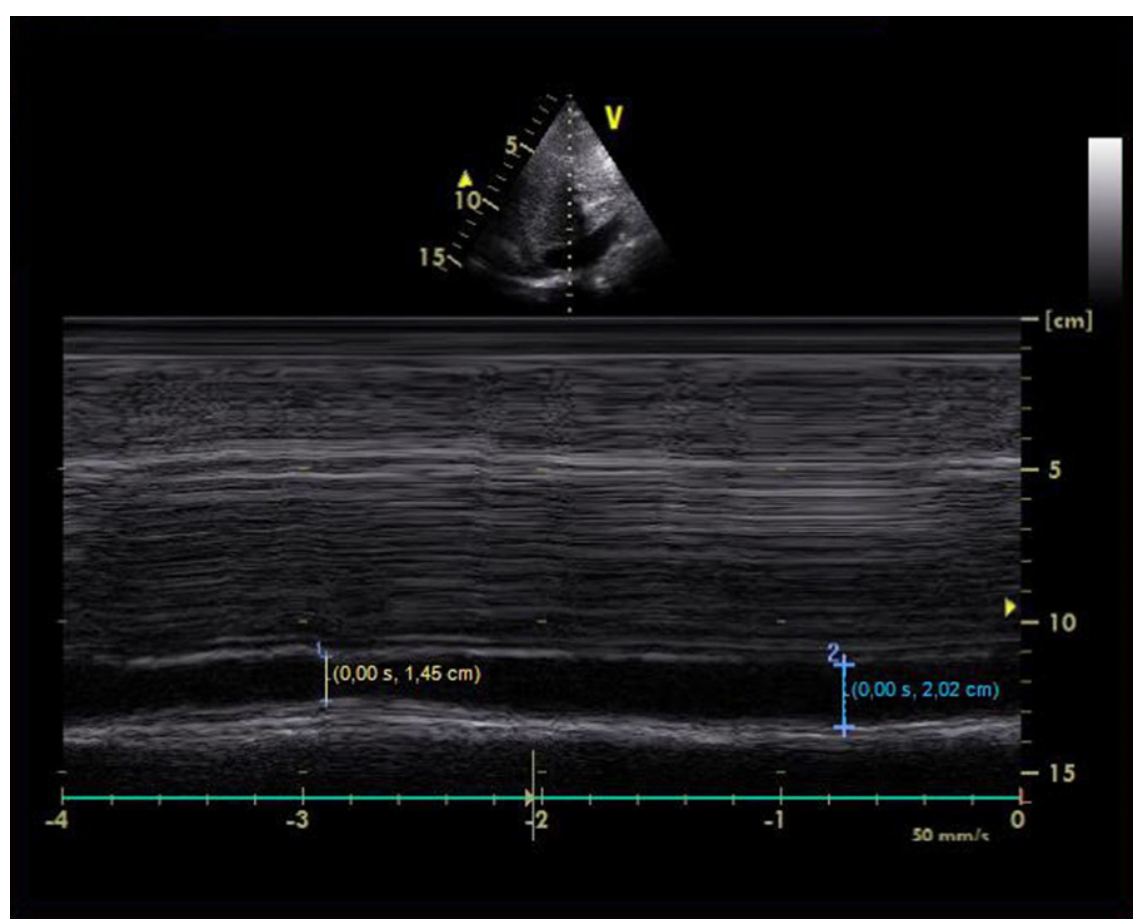

Abb. 1 \ Darstellung der atemabhängigen Schwankungen des Diameters der VCImittels Sonographie im B-Mode und im M-Mode in der langen Achse. Euvolämischer Zustand. $\mathrm{VCICl}=28 \%$

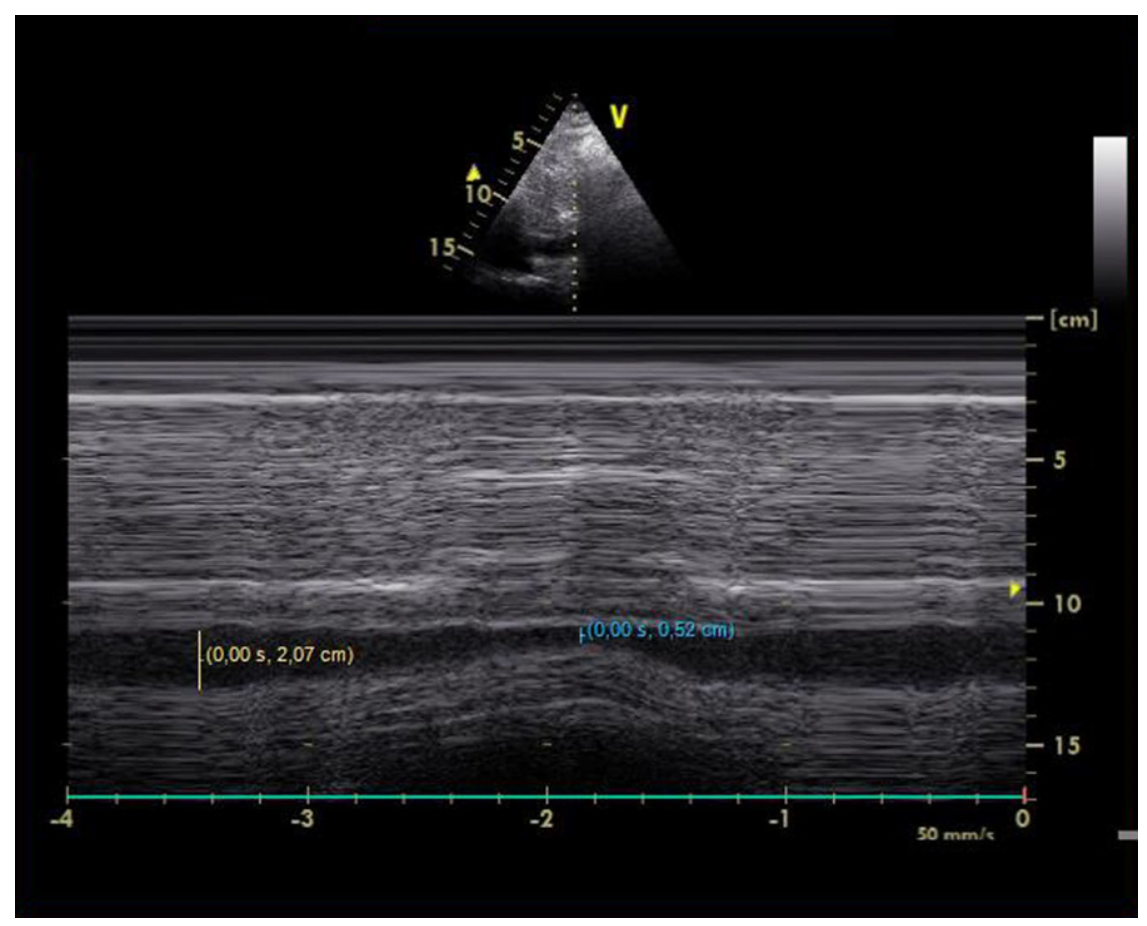

Abb. $2 \Delta$ Darstellung der atemabhängigen Veränderungen des Diameters der VCImittels Sonographie im B-Mode und im M-Mode in der langen Achse. Hypovolämischer Zustand. $\mathrm{VCICI}=75 \%$

\section{Statistik}

Die statistische Analyse erfolgte mit der SPSS ${ }^{\circledR}$ Statistical Package of the Social Sciences (IBM Corp. Released 2017. IBM SPSS Statistics for MAC OS Version 25.0. Armonk, NY, IBM Corp.) und GraphPad Prism Version 8.3.1 for MAC OS (GraphPad Software, San Diego, California USA). Bei ordinal skalierten Merkmalen wurde der exakte Test nach Fisher, bei metrisch skalierten Merkmalen wurden der nichtparametrische Test nach Mann-Whitney und die Spearman-Korrelation verwendet. Zur Prüfung des Zusammenhangs zwischen einer CINB und potenziellen Risikofaktoren wurde eine logistische Regressionsanalyse durchgeführt. Das Signifikanzniveau (SN) wurde bei $p<0,05$ und das Konfidenzintervall (KI) bei $95 \%$ festgelegt.

\section{Ergebnisse}

\section{Demografische und biometrische Daten}

Die Studie umfasste 103 Teilnehmer, 74 Westeuropäer, 11 Türken, 5 Araber und 13 Osteuropäer. Bei allen Studienteilnehmern wurde die Ethnizität erfragt, da unterschiedliche Ergebnisse in Bezug auf den Zusammenhang zwischen CysC-Spiegeln und ethnischer Herkunft gezeigt wurden (auf der einen Seite Farbige und Mexikaner mit niedrigeren CysC-Spiegeln, auf der anderen Seite aber farbige Patienten mit höheren CysC-Spiegeln). In unserer Studie waren alle Probanden hellhäutig, sodass eine Differenzierung hellhäutig vs. farbig nicht möglich war. Es wurden 65 (63,10\%) männliche 38 (36,89\%) weibliche Patienten untersucht. Bei 29 (28,16\%) Patienten kam es im Verlauf zu einer CINB. Davon waren insgesamt 8 ev Patienten (7,77\%) und 21 hv Patienten (20,39\%) betroffen (- Abb. 3). Am ersten Tag nach der HKU stieg das CysC bei 6 (12,24\%) ev und 14 $(25,92 \%)$ hv Patienten und am zweiten Tag bei 2 (4,08\%) ev und 7 (12,96\%) hv Patienten entsprechend einer CINB an. Es bestand ein statistisch signifikanter Zusammenhang zwischen einer CINB und dem Volumenstatus $(p=0,015) ; \mathrm{RR}=2,382$ $(95 \%-\mathrm{KI}=1,163-4,877)(p=0,015)$. Neun $(8,74 \%)$ ev und $7(6,81 \%)$ hv Patienten entwickelten eine CIN $(p=0,587)$ die nach 


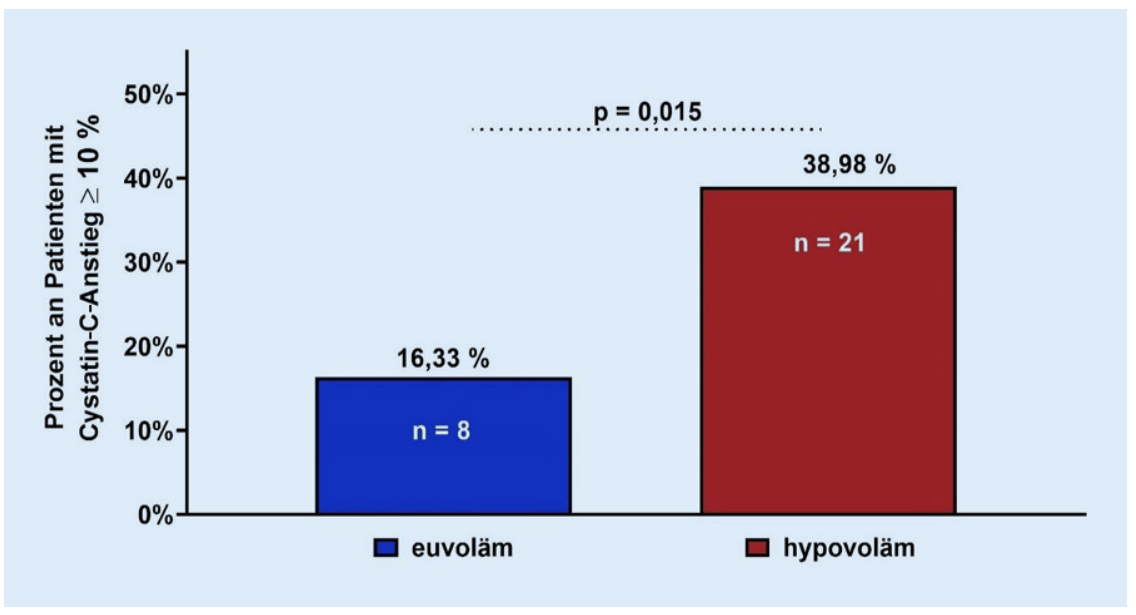

Abb. 3 \ Prozentualer Anteil der Patienten, deren Cystatin-C-Wert im jeweiligen Kollektiv im beobachteten Zeitraum um $\geq 10 \%$ des Ausgangswertes gestiegen ist, $16(24,62 \%)$ männliche und 13 $(34,21 \%)$ weibliche Patienten erkrankten an einer $\operatorname{CINB}(p=0,365)$. Ihr Alter lag bei $68,72 \pm 12,36 \mathrm{Jah}$ ren $(\min =45 ; \max =88$ Jahre). Obwohl das Alter der Patienten keinen prädiktiven Einfluss auf eine CINB hatte, korrelierte das Alter signifikant mit den CysC-Werten, am zweiten Tag sogar stärker als am ersten Tag $\left(\mathrm{r}_{\mathrm{sp}}=0,601 ; p=0,0006\right.$ vs. $\left.\mathrm{r}_{\mathrm{sp}}=0,752 ; p<0,0001\right)$ ( $\bullet$ Abb. 4 zeigt Prozent an Patienten mit einem Cystatin-C-Anstieg $\geq 10 \%$ )

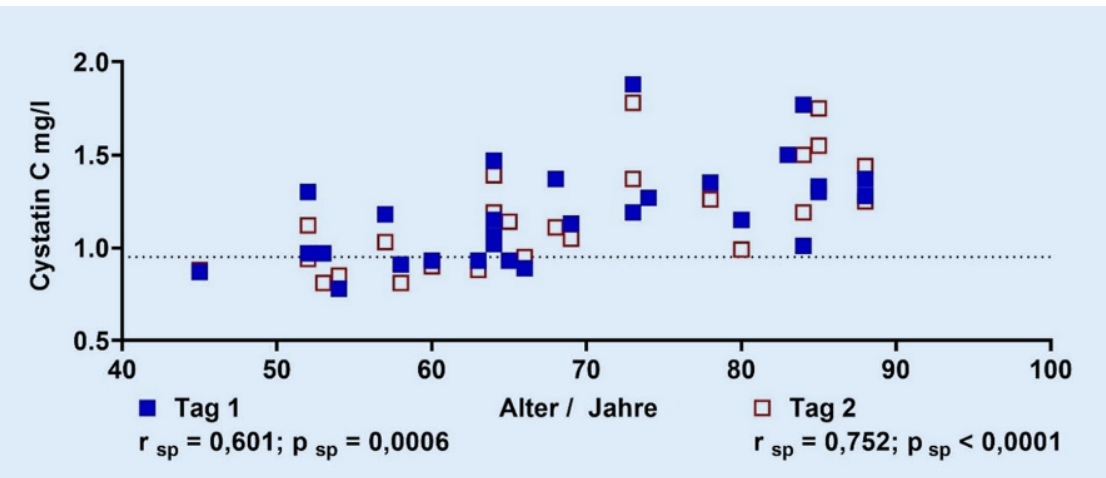

Abb. 4 ॥ Spearman-Korrelation zwischen CysC und dem Alter der CINB(+)-Patienten am ersten und zweiten Tag nach der HK. Gepunktete Linie Laborreferenzwert

der Empfehlung der KDIGO durch einen Kreatininanstieg $>0,3 \mathrm{mg} 48 \mathrm{~h}$ nach $\mathrm{KM}$ Exposition definiert ist [13].

\section{Komorbiditäten}

Als weitere Krankheitsbilder wurden unter anderem die arterielle Hypertonie (aHTN), die koronare Herzkrankheit (KHK) und der Diabetes mellitus Typ 2 (DM2) erfasst. Es konnte kein signifikanter Zusammenhang zwischen diesen Erkrankungen und der CINB hergestellt werden (- Tab. 2). Auch die Adipositas wurde als Krankheitsentität in die vorliegende Analyse mit einbezogen.

\section{Chronische Herzinsuffizienz}

Es erkrankten $28(27,18 \%)$ der Patienten, bei denen eine chronische Herzinsuffizienz (CHF) nach den NYHA-Kriterien feststellbar war, an einer CINB. Die Ergebnisse der Regressionsanalyse deuten darauf hin, dass die CHF einen prädiktiven Einfluss auf die CINB hat, OR=15,099 (95\%$\mathrm{KI}=1,580-144,216)(p=0,018)(\bullet$ Abb. 5).

Als objektives Maß für die CHF wurde bei 86 Patienten der proBNP(, brain natriuretic peptide")-Wert im Serum analysiert. Sowohl bei ev als auch bei hv Patienten korrelierte das CysC bereits vor der HKU mit den proBNP-Werten. Im ev Kollektiv: $r=0,508(p<0,0001)$ und im hv Kollektiv: $r=0,444(p=0,002)$ (ब Abb. 6). Es konnte für keinen Messzeitpunkt eine signifikante Korrelation für proBNP und Krea ermittelt werden.

\section{Vorhofflimmern}

Kardioembolische Ereignisse, die durch ein Vorhofflimmern (VHF) ausgelöst werden, gehören zu den häufigsten Ursachen für Niereninfarkte [20]; 15 (48,38\%) Patienten, die im Verlauf an einer CINB erkrankten, hatten häufig auch ein VHF $(p=0,004)$, sodass sich für diese Patienten ein erhöhtes Risiko für eine CINB ergab: $R R=2,488(95 \%-K I=1,373-4,508)$ $(p=0,003)(\bullet$ Abb. 7$)$.

\section{Adipositas}

Die Adipositas stellt nicht nur einen Risikofaktor für kardiovaskuläre Erkrankungen dar, sondern kann auch zu Nierenfunktionsstörungen führen $[9,14] ; 13(43,33 \%)$ Patienten mit einem BMI $\left(B M l>30 \mathrm{~kg} / \mathrm{m}^{2}\right)$ erkrankten an einer CINB. Die Regressionsanalyse zeigte, dass ein prädiktiver Zusammenhang zwischen der Adipositas und der CINB besteht, $O R=4,326$ $(95 \%-K I=1,281-14,602) \quad(p=0,018)$; Patienten mit einer Adipositas hatten ein erhöhtes Risiko, an einer CINB zu erkranken: $\quad \mathrm{RR}=2,166 \quad(95 \%-\mathrm{Kl}=1,163-4,036)$ $(p=0,014)$ (ब Abb. 8).

Die CysC-Werte korrelierten bereits vor der HKU leicht, aber dennoch signifikant mit den BMI-Werten der Patienten des Gesamtkollektivs: $\mathrm{r}=0,218 \quad(p=0,031)$ (- Abb. 9). Innerhalb der jeweiligen Vergleichsgruppen (ev vs. hv) konnte keine signifikante Korrelation ermittelt werden.

\section{Medikamente}

Mehr als $50 \%$ der Patienten, die an einer CINB erkrankten, nahmen Schleifendiuretika in unterschiedlicher Dosierung ein, $n=17$ (58,62\%). Die Ergebnisse lassen einen signifikanten Zusammenhang zwischen der Einnahme von Diuretika und der CINB erkennen $(p=0,044) ; \mathrm{RR}=1,976$ $(95 \%-\mathrm{KI}=1,056-3,699) \quad(p=0,033)$. Es konnte kein erhöhtes Risiko für eine CINB durch die Einnahme weiterer potenziell nephrotoxischer Medikamente einschließlich NSAR $(n=4)$ und Metformin $(n=4)$ festgestellt werden (• Tab. 2). 


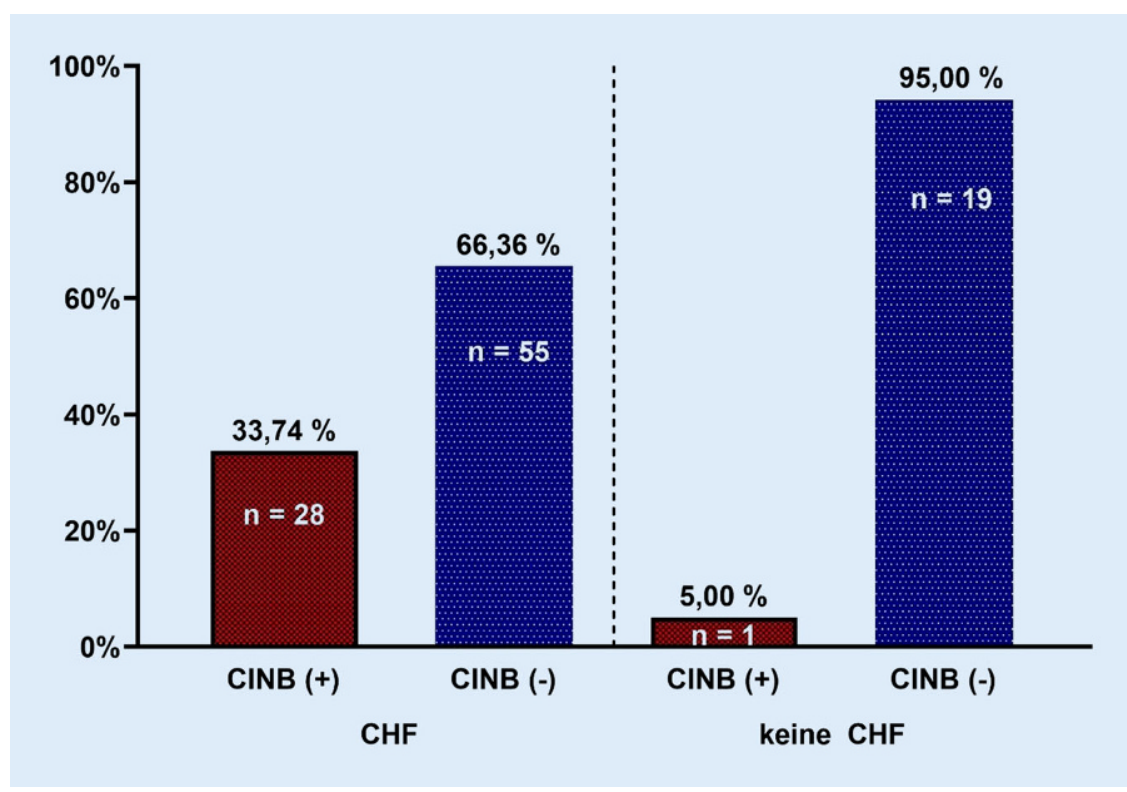

Abb. $5 \Delta$ Prozentuale Verteilung CINB ( \pm )-Patienten mit und ohne CHF

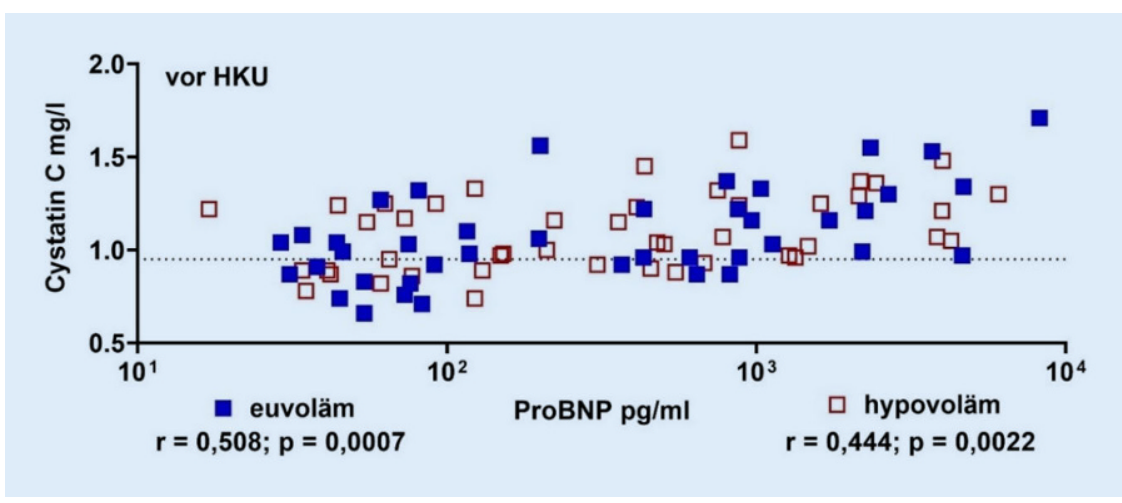

Abb. 6 \ Spearman-Korrelation zwischen CysC und proBNPvor HKU. Gepunktete Linie Laborreferenzwert

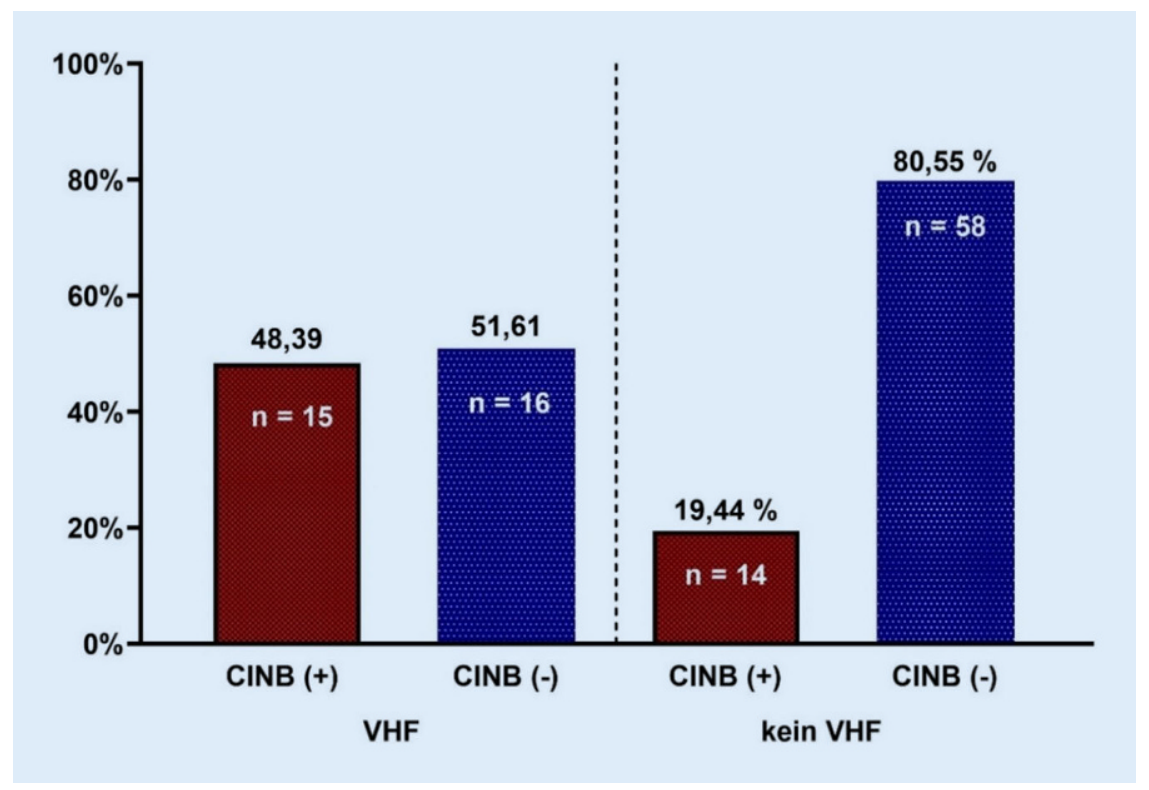

Abb. 7 A Prozentuale Verteilung CINB( \pm )-Patienten mit und ohne VHF

\section{Kontrastmittelmenge}

Bei der HKU wurde das Kontrastmittel Iomeprol (Imeron 350 Bracco Imaging Deutschland $\mathrm{GmbH}$ ) verwendet.

Ev CINB (+)-Patienten erhielten im Median $55,00 \mathrm{ml}$ JKM $(\min =15,00 \mathrm{ml}$; $\max =160,00 \mathrm{ml}$ ), ev CINB (-)-Patienten im Median $50,00 \mathrm{ml}(\mathrm{min}=13,00 \mathrm{ml}$; $\max =160,00 \mathrm{ml})(p=0,754)$. Hv CINB (+)Patienten wurden im Median $30,00 \mathrm{ml}$ $(\min =17,00 \mathrm{ml} ; \max =260,00 \mathrm{ml})$ verabreicht. Bei den hv CINB (-)-Patienten lag der Median des injizierten Kontrastmittels bei $50,00 \mathrm{ml}(\min =7,00 \mathrm{ml} ; \max =260 \mathrm{ml})$ $(p=0,116)$.

Die CysC-Werte ev CINB (+)-Patienten korrelierten am zweiten Tag nach der HKU signifikant mit der verabreichten Kontrastmittelmenge $(r=0,910 ; p=0,003)$. Im hv Kollektiv konnte keine Korrelation festgestellt werden. Hypovoläme CINB (+)-Patienten haben möglicherweise bereits eine erhöhte Suszeptibilität für einen CysCAnstieg, sodass eine geringere JKM-Dosierung ausreicht, um eine CINB hervorzurufen.

\section{Diskussion}

Der Einsatz von JKM ist in der interventionellen Kardiologie, nicht selten alternativlos. Der initiale Volumenstatus der $\mathrm{Pa}$ tienten wurde in den bisherigen Untersuchungen zur CIN kaum beachtet.

Ein hypovolämischer Hydratationszustand alleine kann durch Exprimierung proinflammatorischer Mediatoren zu Tubulusschäden führen. Der Pathomechanismus beruht auf der Hyperosmolarität des zirkulierenden Blutvolumens, das zu einer gesteigerten Ausschüttung von Vasopressin aus dem Hypophysenhinterlappen und einer vermehrten Synthese der Aldose-Reduktase in den Tubulusepithelzellen beiträgt. Die Aldose-Reduktase ist ein Enzym, das bei chronischer Dehydratation zu einer vermehrten Bildung von Harnsäure und damit intrarenal zu einem gesteigerten oxidativen Stress, Endotheldysfunktion und der Ausschüttung proinflammatorischer Mediatoren führt [22].

Lau et al. kamen anhand ihrer tierexperimentellen Daten zu dem Ergebnis, dass nach JKM-Applikation bei hypovolämen Mäusen es zu einer nachweislichen Akku- 


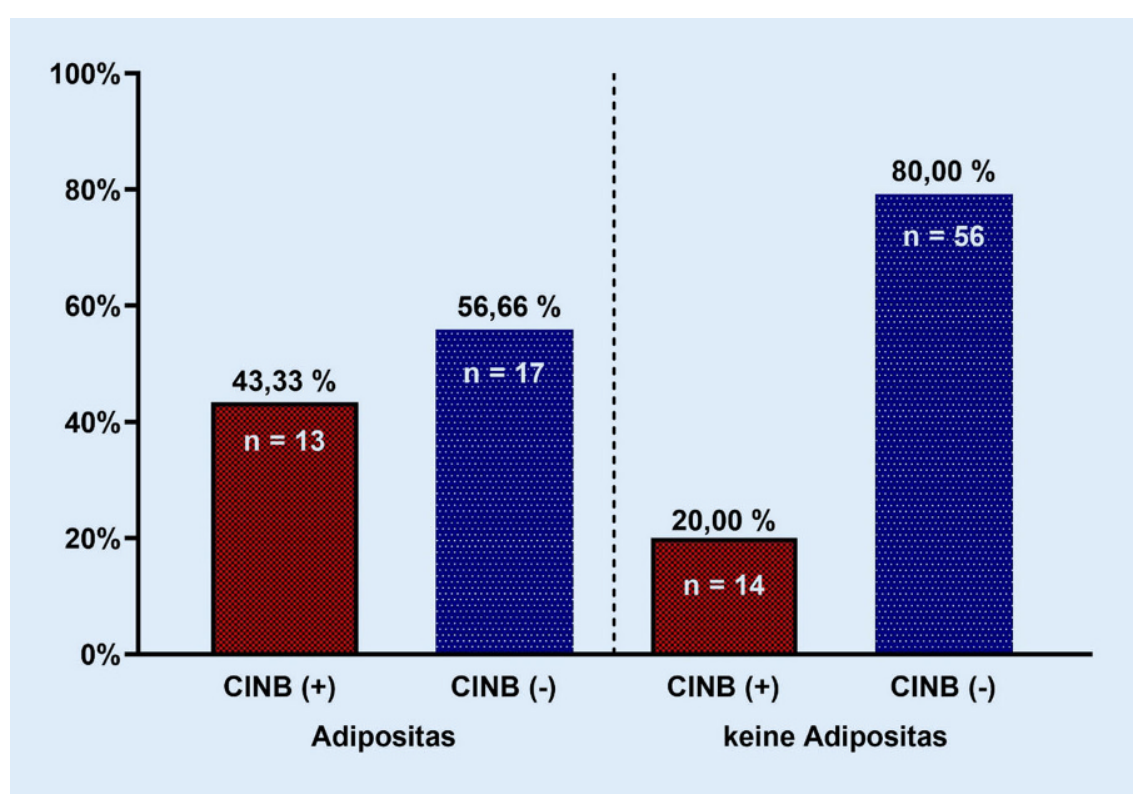

Abb. $8 \Delta$ Prozentuale Verteilung CINB ( \pm )-Patienten mit und ohne Adipositas (BMl>30 kg/m²)

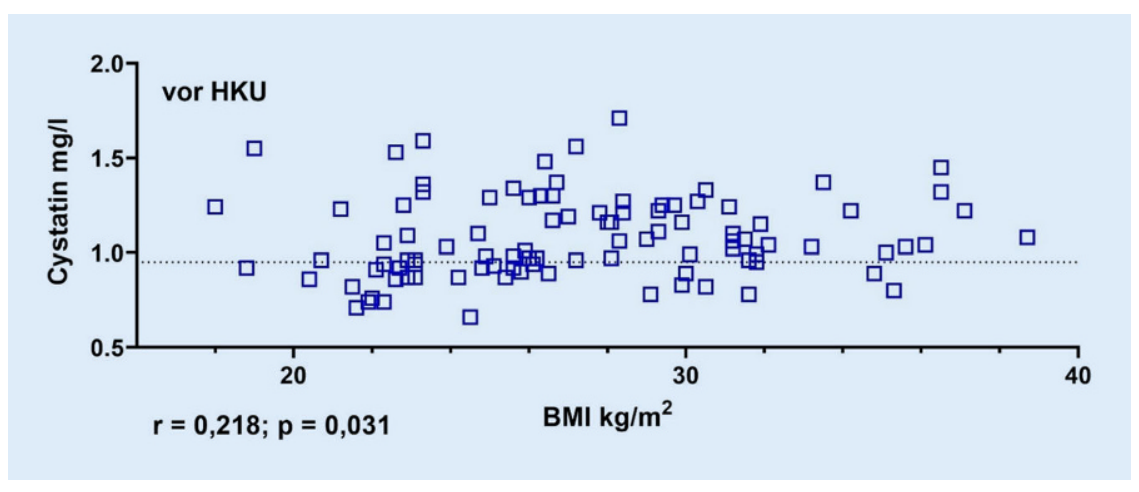

Abb. 9 \ Spearman-Korrelation zwischen CysC und BMlim Gesamtkollektiv vor HKU. Gepunktete Linie Laborreferenzwert

mulation von JKM in den Tubuli kommt. Bei Volumenmangel zeigt das Enzym Dipeptidase 1 am Bürstensaum der Tubulusepithelzellen eine gesteigerte Aktivität bei der aktiven Resorption von Kontrastmittel durch das Tubulusepithel, wodurch eine Aktivierung des NLPR3-Inflammasoms durch Phagozyten und Rekrutierung von Leukozyten durch IL-1 $\beta$ stattfindet. Dadurch wird eine entzündliche Immunantwort im Umfeld der Tubuluszellen unterhalten, die zu Schäden am Tubulusepithel führt [16].

Es muss daher überlegt werden, dass möglicherweise nicht die KM-Applikation per se, sondern die KM-Applikation in Kombination mit einem hypovolämen Volumenstatus mit einem erhöhten Risiko für eine Nierenfunktionsstörung einhergeht.
Nierenfunktionsstörung anhand der Serumcystatin-C-Werte

Dass durch die Verwendung des CysC auch in dieser Studie eine deutlich höhere Rate akuter Nierenfunktionsstörung nachgewiesen wurde als mit der Bestimmung der Krea-Werte, unterstreicht damit die Diskussion, dass die kreatininbasierte Definition nicht optimal geeignet scheint, um eine CIN nach KM-Exposition zuverlässig zu detektieren [5]. Diskutiert wird, dass das Cys C möglicherweise schon vor KM-Exposition den besseren Parameter darstellt, Patienten mit einem erhöhten Risiko für eine $\mathrm{CIN}(\mathrm{B}) \mathrm{zu}$ identifizieren. Nozue et al. zeigten in einer Untersuchung, dass ein präexpositioneller CysC-Cut-off-Wert von $1,26 \mathrm{mg} / \mathrm{l}$ mit einer Sensitivität von $75 \%$ und Spezifität von $73 \%$ ein CIN vorhersagen kann [15].

Bei 7 von 16 an CIN erkrankten Patienten lag das CysC über und bei 9 Patienten unterhalb dieses Cut-off Werts, sodass sich eine Sensitivität von $43,75 \%$ und eine Spezifität von $83,91 \%$ ergaben. Somit sollte auch der von Nozue et al. definierteCut-offWert von 1,26 mg/dl möglicherweise noch nicht als endgültig betrachtet werden, sondern Anlass geben, um den optimalen Cutoff-Wert anhand weiterer Studien zu evaluieren und ggf. weiter anzupassen.

\section{Vorhofflimmern}

Im Jahr 2016 erkannten Ballı et al., dass VHF bei Patienten mit STEMI ein unabhängiger Prädiktor für eine kreatininbasierte CIN ist: $\mathrm{OR}=6,945 \quad(95 \%-\mathrm{KI}=2,789-17,293)$. Ein ähnlicher Zusammenhang wurde auch bei den Patienten in der vorliegenden Studie festgestellt: OR=6,606 (95\%$\mathrm{Kl}=1,909-22,861)$. Wang et al. konnten nachweisen, dass bei bis zu $52 \%$ der in ihrer Studie untersuchten Patienten die Dosierung der oralen Antikoagulation angepasst werden musste, nachdem die eGFR mit Serumcystatin $C$ anstelle von Kreatinin bestimmt worden war [24].

\section{Chronische Herzinsuffizienz}

Im Vergleich zu der kreatininbasierten Definition einer CIN deuten die Ergebnisse darauf hin, dass bei Patienten, die an einer CINB erkrankten, ein signifikanter prädiktiver Zusammenhang zwischen der $\mathrm{CHF}$ und der CINB besteht $(p=0,011)$. Das CysC korreliert bereits vor der HKU mit den proBNPWerten, wohingegen keine Korrelation für proBNP und Krea ermittelt werden konnte. Dupont et al. zeigten, dass eine Kombination der Parameter Serumcystatin $C$ und proBNP sich hervorragend zur Risikostratifikation bei der Vorhersage einer MACE ("major adverse cardiovascular event") bei Patienten mit stabilen CHF eignet [7].

\section{Adipositas}

Knight et al. wiesen in ihrer Arbeit darauf hin, dass nach Anpassung an die Nierenfunktion anhand der Kea-Clearence Adipositas als unabhängiger Faktor zu erhöhten CysC-Werten beiträgt [15]. Interessanter- 
weise zeigte CysC in diesem Zusammenhang eine protektive Wirkung gegenüber inflammatorischen Prozessen an insulinsensitiven Zellen [7]. Auch in der vorliegenden Studie korrelieren die BMI-Werte vor der HKU mit den Cyst-Werten. Kabeer et al. erkannten, dass ein $\mathrm{BMI}>30 \mathrm{~kg} / \mathrm{m}^{2}$ ein Risikofaktor für eine Krea-basierte CIN darstellt [12]. Ein solcher Zusammenhang konnte für die Krea-basierte Definition der $\mathrm{CIN}$ in dieser Studie nicht hergestellt werden. Die Ergebnisse deuten jedoch darauf hin, dass adipöse Patienten ein erhöhtes Risiko für die CINB haben.

\section{Diuretika}

Die Einnahme von Schleifendiuretika war mit einem erhöhten Risiko für eine CINB verbunden. In früheren Studien wurde über die potenzielle nephrotoxische Wirkung von Schleifendiuretika berichtet. Die Azidurie steht dabei unter dem Verdacht, die Bildung von freien Radikalen infolge einer KM-Exposition - und dadurch Nephrotoxizität - zu verstärken [18, 21]. Majumdar et al. empfahlen Patienten mit chronischer Niereninsuffizienz, Furosemid vor der Verabreichung von JKM im Rahmen einer HKU zu pausieren [12].

\section{Limitationen}

\section{Erfassung des Volumenstatus}

Die sonographische Darstellung der VCl ist eine etablierte Methode zur Bestimmung des Volumenstatus. Faktoren wie Trikuspidal- oder Pulmonalklappenvitien, erhöhter pulmonalarterieller Widerstand, fibrotische Prozesse der Leber, die Expertise des Untersuchers und Compliance des Patienten können die Messergebnisse beeinflussen [2].

Die Arbeitsgruppe für kardiovaskuläre Intensiv- und Notfallmedizin der DGK empfiehlt Richtwerte für den VCl-Durchmesser, durch die auf den Volumenstatus geschlossen werden kann [10].

\section{Patienten mit chronischer Niereninsuffizienz}

Eine Analyse zur Auswirkung einer Hypovolämie bei Patienten, die bereits erhöhte Krea-Werte zeigten, wurde in dieser Arbeit nicht untersucht. Viele Patienten mit fortgeschrittener kardialer Grunderkrankung weisen jedoch eine zusätzliche Nierenfunktionsstörung auf. Ob bei diesen Patienten eine zusätzliche Hypovolämie zu einem frühzeitigeren Cystatin-C-Anstieg beiträgt, könnte Gegenstand weiterer Untersuchungen sein.

\section{Fazit für die Praxis}

- Eine CIN nach JKM-Exposition ist häufiger, wenn man der Definition eine CysCErhöhung $\geq 10 \%$ des Ausgangswertes zugrunde legt, als wenn man die Kreabasierte Definition verwendet.

- Ein hypovolämer Volumenstatus ist ein Risikofaktor für eine CIN nach KM-Exposition, wenn diese anhand eines CysCAnstiegs $\geq 10 \%$ des Ausgangswertes definiert wird.

- Sowohl die CHF als auch VHF stellen neben der Adipositas mögliche prädiktive Faktoren für ein erhöhtes Risiko einer CIN dar, wenn die Definition der akuten CIN auf dem Verlauf der CysC-Werte basiert und nicht der Bestimmung der Krea-Werte.

- Medikamentöse Therapie müsste ggf. anders gesteuert werden, wenn man als Nierenfunktionsparameter Serumcystatin C anstelle von Kreatinin nutzt.

- Periprozedural kann das Absetzen einer diuretischen Medikation um die Zeit der KM-Applikation das Risiko für eine CINB möglicherweise reduzieren.

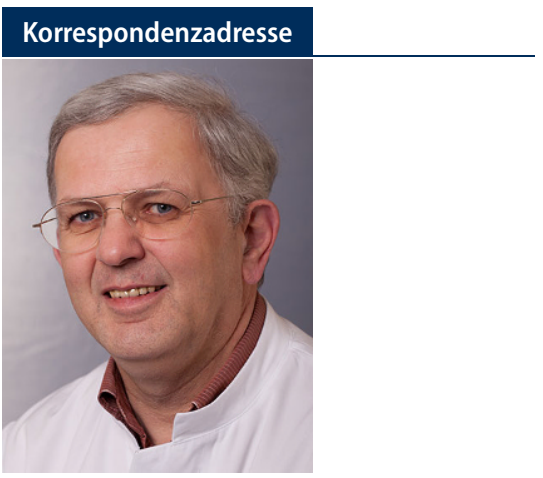

Univ.-Prof. Dr. med. Hans-Joachim Trappe Medizinische Klinik II (Schwerpunkte Kardiologie und Angiologie), Marien Hospital Herne Universitätsklinik, Ruhr-Universität Bochum

Hölkeskampring 40, 44625 Herne, Deutschland Hans-Joachim.Trappe@ruhr-uni-bochum.de

Funding. Open Access funding enabled and organized by Projekt DEAL.

\section{Einhaltung ethischer Richtlinien}

Interessenkonflikt. F.M. Vahed und H.-J. Trappe geben an, dass kein Interessenkonflikt besteht.

Alle beschriebenen Untersuchungen am Menschen oder an menschlichem Gewebe wurden mit Zustimmung der zuständigen Ethikkommission, im Einklang mit nationalem Recht sowie gemäß der Deklaration von Helsinki von 1975 (in der aktuellen, überarbeiteten Fassung) durchgeführt. Von allen beteiligten Patienten liegt eine Einverständniserklärung vor.

Open Access. Dieser Artikel wird unter der Creative Commons Namensnennung 4.0 International Lizenz veröffentlicht, welche die Nutzung, Vervielfältigung, Bearbeitung, Verbreitung und Wiedergabe in jeglichem Medium und Format erlaubt, sofern Sie den/die ursprünglichen Autor(en) und die Quelle ordnungsgemäß nennen, einen Link zur Creative Commons Lizenz beifügen und angeben, ob Änderungen vorgenommen wurden.

Die in diesem Artikel enthaltenen Bilder und sonstiges Drittmaterial unterliegen ebenfalls der genannten Creative Commons Lizenz, sofern sich aus der Abbildungslegende nichts anderes ergibt. Sofern das betreffende Material nicht unter der genannten Creative Commons Lizenz steht und die betreffende Handlung nicht nach gesetzlichen Vorschriften erlaubt ist, ist für die oben aufgeführten Weiterverwendungen des Materials die Einwilligung des jeweiligen Rechteinhabers einzuholen.

Weitere Details zur Lizenz entnehmen Sie bitte der Lizenzinformation auf http://creativecommons.org/ licenses/by/4.0/deed.de.

\section{Literatur}

\section{Verwendete Literatur}

1. Beigel R, Cercek B, Luo H et al (2013) Noninvasive evaluation of right atrial pressure. J Am Soc Echocardiogr 26:1033-1042

2. Blehar DJ, Resop D, Chin B et al (2012) Inferior vena cava displacement during respirophasic ultrasound imaging. Crit Ultrasound J 4:18

3. Briguori C, Visconti G, Rivera NV et al (2010) Cystatin $C$ and contrast-induced acute kidney injury. Circulation 121:2117-2122

4. Brown JR, Mackenzie TA, Maddox TM et al (2015) Acute kidney injury risk prediction in patients undergoing coronary angiography in a national veterans health administration cohort with external validation. JAHA4:1-15

5. Caplin B, Yang C-W, Anand S et al (2019) The international society of nephrology international consortium of collaborators on chronic kidney disease of unknown etiology: report of the working group on approaches to population-level detection strategies and recommendations for minimum dataset. Kidney Int 95:4-10

6. D'Amore C, Nuzzo S, Briguori C (2020) Biomarkers of contrast-induced nephropathy: which ones are clinically important? Interv Cardiol Clin 9:335-344

7. Dedual MA, Wueest S, Challa TD et al (2020) Obesity-induced increase in cystatin $C$ alleviates tissue inflammation. Diabetes 69:1927-1935

8. Dharnidharka VR, Kwon C, Stevens G (2002) Serum cystatin $C$ is superior to serum creatinine as 
a marker of kidney function: a meta-analysis. Am J Kidney Dis 40:221-226

9. Eckel N, Li Y, Kuxhaus $O$ et al (2018) Transition from metabolic healthy to unhealthy phenotypes and association with cardiovascular disease risk across BMI categories in 90.257 women (the nurses' health study): 30 year follow-up from a prospective cohort study. Lancet Diabetes Endocrinol 6:714-724

10. Janssens $U$, Jung $C$, Hennersdorf M et al (2016) Empfehlungen zum hämodynamischen Monitoring in der internistischen Intensivmedizin. Kardiologe 10:149-169

11. Jeffrey RB, Federle MP (1988) The collapsed inferior vena cava: CT evidence of hypovolemia. AJR Am J Roentgenol 150:431-432

12. Kabeer MA, Cross J, Hamilton G et al (2021) Obesity as a risk factor for radiographic contrast-induced nephropathy. Angiology 72:274-278

13. Kdigo (2012) Clinical practice guideline for acute kidney injury section 4 : contrast-induced AKI. Kidney Int Suppl 2:69-88

14. Ke B, Shen W, Fang X et al (2018) The NLPR3 inflammasome and obesity-related kidney disease. J Cell Mol Med 22:16-24

15. Knight EL, Verhave JC, Spiegelman D et al (2004) Factors influencing serum cystatin $C$ levels other than renal function and the impact on renal function measurement. Kidney Int 65:1416-1421

16. Latus J, Schwenger V, Schlieper G et al (2020) Kontrastmittelinduzierte akute Nierenschädigung - Konsensuspapier der Arbeitsgemeinschaft „Herz - Niere" der Deutschen Gesellschaft für Kardiologie - Herz- und Kreislaufforschung e.V. und der Deutschen Gesellschaft für Nephrologie e. V. Kardiologe 14:494-504

17. Mehran R, Nikolsky E (2006) Contrast-induced nephropathy: definition, epidemiology, and patients at risk. Kidney Int Suppl 100:11-15

18. Nagdev AD, Merchant RC, Tirado-Gonzalez A et al (2010) Emergency department bedside ultrasonographic measurement of the caval index for noninvasive determination of low central venous pressure. Ann Emerg Med 55:290-295

19. Nash K, Hafeez A, Hou S (2002) Hospital-acquired renal insufficiency. Am J Kidney Dis 39:930-936

20. Oh YK, Yang CW, Kim YL et al (2016) Clinical characteristics and outcomes of renal infarction. Am J Kidney Dis 67:243-250

21. Rihal CS, Textor SC, Grill DE et al (2002) Incidence and prognostic importance of acute renal failure after percutaneous coronary intervention. Circulation 105:2259-2264

22. Ruepp B, Bohren KM, Gabbay KH (1996) Characterization of the osmotic response element of the human aldose reductase gene promoter. Proc Natl Acad SciUSA 93:8624-8629

23. Stawicki SP, Braslow BM, Panebianco NLet al (2009) Intensivist use of hand-carried ultrasonography to measure IVC collapsibility in estimating intravascular volume status: correlations with CVP. J Am Coll Surg 209:55-61

24. Wang CH, Rubinsky AD, Minichiello T et al (2018) Creatinine versus cystatin C: differing estimates of renal function in hospitalized veterans receiving anticoagulants. J Gen Intern Med 33:1299-1306

25. Weisbord SD, Chen H, Stone RA et al (2006) Associations of increases in serum creatinine with mortality and length of hospital stay after coronary angiography.J Am Soc Nephrol 17:2871-2877
Contrast-induced acute kidney injury measured by serum cystatin C. Comparison between euvolemic and hypovolemic patients after coronary angiography

Abstract: The significance of contrast-induced renal impairment is unclear. This prospective study investigates the influence of hypovolemia on cystatin $C$ values after contrast agent exposure during cardiac catheterization.

Patients and methods: A total of 103 patients were enrolled of which 49 were euvolemic and 54 were hypovolemic ( $68.72 \pm 12.36$ years, $63.10 \%$ male). Intravascular volume status was determined by ultrasound-assisted measurement of the collapsibility of the inferior vena cava. Cystatin C values were determined before, on the first and second day after cardiac catheterization. A 10\% increase from baseline was declared as CINB.

Results: In 8 (16.33\%) patients with euvolemia and 21 (38.89\%) patients with hypovolemia CINB developed $(p=0.015)$. The relative risk (RR) for CINB in hypovolemic patients was $2.38(95 \% \mathrm{Cl}=1.163-4.877, p=0.015)$. Atrial fibrillation (AF) was also a risk factor for $\mathrm{CINB}$ : $\mathrm{RR}=2.488(95 \% \mathrm{Cl}=1.373-4.508, p=0.003) ; \mathrm{OR}=6.606(95 \%$ $\mathrm{Cl}=1.909-22.861)$. Obese patients with a $\mathrm{BMI}>30 \mathrm{~kg} / \mathrm{m}^{2}$ had an increased risk of developing $\mathrm{CINB}$ : $\mathrm{OR}=4.326(95 \% \mathrm{Cl}=1.281-14.602, p=0.018) \mathrm{RR}=2.166(95 \%$ $\mathrm{Cl}=1.163-4.036, p=0.014)$. Patients taking loop diuretics also had an increased risk for CINB: $\mathrm{RR}=1.976(95 \% \mathrm{Cl}=1.056-3.699, p=0.033)$. Chronic heart failure (CHF) was a predictive factor for CINB: $\mathrm{OR}=13.27(95 \% \mathrm{Cl}=1.453-121.199, p=0.018)$.

Conclusion: Defining acute renal impairment using a serum cystatin $C$ increase $>10 \%$, hypovolemic patients have an increased risk of CIN following contrast agent exposure than euvolemic patients. In addition, AF, CHF and the use of diuretics are associated with an increased risk of CINB.

\section{Keywords}

Cystatin C · Contrast-induced nephropathy · Contrast agent · Volume status · Cardiac catheterization

\section{Weiterführende Literatur}

26. Ballı M, Taşolar H, Çetin M et al (2016) Is atrial fibrillation a risk factor for contrast-induced nephropathy in patients with ST-elevation myocardial infarction? J Cardiol 67:327-330

27. Dupont M, Wu Y, Hazen SL et al (2012) Cystatin C identifies patients with stable chronic heart failure at increased risk for adverse cardiovascular events. Circ Heart Fail 5:602-609

28. Levi TM, Rocha MS, Almeida DN et al (2012) Furosemide is associated with acute kidney injury in critically ill patients. Braz J Med Biol Res 45:827-833

29. Majumdar SR, Kjellstrand CM, Tymchak WJ et al (2009) Forced euvolemic diuresis with mannitol and furosemide for prevention of contrast-induced nephropathy in patients with CKD undergoing coronary angiography: a randomized controlled trial. Am J Kidney Dis 54:602-609

30. Nozue T, Michishita I, Mizuguchi I (2010) Predictive value of serum cystatin $C, \beta 2$-microglobulin, and urinary liver-type fatty acid-binding protein on the development of contrast-induced nephropathy. Cardiovasc Interv Ther 25:85-90

31. Solomon R, Werner C, Mann D et al (1994) Effects of saline, mannitol, and furosemide on acute decreases in renal function induced by radiocontrast agents. N Engl J Med 331:1416-1420

32. Weinstein JM, Heyman S, Brezis M (1992) Potential deleterious effect of furosemide in radiocontrast nephropathy. Nephron 62:413-415 